\title{
The Jacobi-Maupertuis Principle in Variational Integrators
}

\author{
Sujit Nair*, Sina Ober-Blöbaum ${ }^{\dagger}$ and Jerrold E. Marsden* \\ ${ }^{*}$ Control and Dynamical Systems, California Institute of Technology 107-81, Pasadena, CA 91125 \\ ${ }^{\dagger}$ Department of Mathematics, University of Paderborn, D-33098 Paderborn, Germany
}

\begin{abstract}
In this paper, we develop a hybrid variational integrator based on the Jacobi-Maupertuis Principle of Least Action. The Jacobi-Maupertuis principle states that for a mechanical system with total energy $E$ and potential energy $V(q)$, the curve traced out by the system on a constant energy surface minimizes the action given by $\int \sqrt{2(E-V(q))} d s$ where $d s$ is the line element on the constant energy surface with respect to the kinetic energy of the system. The key feature is that the principle is a parametrization independent geodesic problem. We show that this principle can be combined with traditional variational integrators and can be used to efficiently handle high velocity regions where small time steps would otherwise be required. This is done by switching between the Hamilton principle and the Jacobi-Maupertuis principle depending upon the kinetic energy of the system. We demonstrate our technique for the Kepler problem and discuss some ongoing and future work in studying the energy and momentum behavior of the resulting integrator.
\end{abstract}

Keywords: variational integrator, discrete mechanics, Jacobi-Maupertius principle

PACS: $02.40 . \mathrm{Yy}, 02.60 . \mathrm{Cb}, 45.10 . \mathrm{Db}$

\section{MATHEMATICAL PRELIMINARIES}

Lagrangian and Hamiltonian Systems. Let $Q$ be a manifold with local coordinates given by $q=\left(q^{i}\right), i=1, \ldots, n$. Let $q(t) \in Q \subseteq \mathbb{R}^{n}$ be a curve with $t \in[0, T]$. Let $T_{q} Q$ and $T_{q}^{*} Q$ be the tangent and cotangent spaces with velocities $\dot{q}(t) \in T_{q} Q \subseteq \mathbb{R}^{n}$ and momenta $p(t) \in T_{q}^{*} Q \subseteq \mathbb{R}^{n}$, respectively. Consider a regular Langrangian $L: T Q \rightarrow \mathbb{R}, L=K-V$, consisting of kinetic energy $K: T Q \rightarrow \mathbb{R}$ minus potential energy $V: Q \rightarrow \mathbb{R}$. Assume that the kinetic energy is given by $K=\frac{1}{2} g_{i j} \dot{q}^{i} \dot{q}^{j}$ with a Riemannian metric $g$. By the Legendre transformation $\mathbb{F} L: T Q \rightarrow T^{*} Q,(q, \dot{q}) \mapsto(q, \partial L / \partial \dot{q})=$ $(q, p)$, the Hamiltonian $H: T^{*} Q \rightarrow \mathbb{R}$ is determined as $H(q, p)=p \dot{q}-L(q, \dot{q})$, where $p=\mathbb{F} L(q, \dot{q})$ defines $\dot{q}$ as a function of $(q, p)$. The equations of motion of a Lagrangian system are given by the Euler-Lagrange equations that are derived via Hamilton's principle. Trajectories have to be stationary solutions of the following action principle $[5,6]$

$$
\delta \int_{0}^{T} L(q, \dot{q}) d t=0
$$

for all variations $\delta q$ with $\delta q(0)=\delta q(T)=0$. This gives the Euler-Lagrange equations

$$
\frac{d}{d t} \frac{\partial}{\partial \dot{q}} L(q, \dot{q})-\frac{\partial}{\partial q} L(q, \dot{q})=0
$$

describing the time evolution of a Lagrangian system. These equations are equivalent to the Hamiltonian equations reading in coordinates as $\dot{q}=\frac{\partial}{\partial p} H(q, p), \dot{p}=-\frac{\partial}{\partial q} H(q, p)$.

The Jacobi-Maupertuis Principle. By the assumption that the Hamiltonian does not explicitly depend on time, i.e. $H(q, p)$, we have an energy conserving system with constant energy $E_{0}$. Thus, solutions can be restricted to the energy surface $E=E_{0}$ leading to the principle of least action of Maupertuis [1,2]. Let $\gamma$ be a curve in $Q$ parametrized by $\tau, a \leq \tau \leq b, \gamma(a)=q_{0}, \gamma(b)=q_{N}$. Then, among all curves $q=\gamma(\tau)$ connecting the two points $q_{0}$ and $q_{N}$ parametrized so that the Hamiltonian function has a fixed value $H(q, p)=E_{0}$, the trajectory of the Hamiltonian equations of dynamics is an extremal of the integral of the action

$$
\int_{\gamma} p d q=\int_{\gamma} p \dot{q} d \tau=\int_{\gamma} \frac{\partial L}{\partial \dot{q}}(\tau) \dot{q}(\tau) d \tau
$$

Note, that the interval $a \leq \tau \leq b$ parametrizing the curve $\gamma$ is not fixed and can be different for different curves whereas the energy must be the same. This energy constant can be used to determine the time of a trajectory (cf. equation (11)) since the proposed principle determines only the shape of a trajectory but not the time.

\footnotetext{
CP1168, Vol. 1, Numerical Analysis and Applied Mathematics, International Conference 2009 edited by T. E. Simos, G. Psihoyios, and Ch. Tsitouras 02009 American Institute of Physics 978-0-7354-0705-3/09/\$25.00
} 
In the presence of potential, the trajectories of the system are geodesics in a certain Riemannian metric. Let $d s^{2}$ be a Riemannian metric on configuration space which gives the kinetic energy (such that $\left.T=\frac{1}{2}(d s / d \tau)^{2}\right)$. In addition let the energy $E_{0}$ be constant and $V(q)<E_{0}$. We then have $L=T-V, H=T+V$ and $(\partial L / \partial \dot{q}) \dot{q}=2 T=(d s / d \tau)^{2}=$ $2\left(E_{0}-V\right)$. The principle stated in (3) results into

$$
\int_{\gamma} \frac{\partial L}{\partial \dot{q}}(\tau) \dot{q}(\tau) d \tau=\int_{\gamma} \sqrt{2\left(E_{0}-V(q)\right)} d s=\sqrt{2} \int_{\gamma} d \rho
$$

with the Riemannian metric $\rho$ defined as $d \rho=\sqrt{2\left(E_{0}-V(q)\right.} d s$. Thus, the trajectories are geodesics in the metric $d \rho$. By substituting $d s^{2}=g_{i j} d q^{i} d q^{j}$ into (4) we are searching for stationary points of

$$
\int_{\gamma} \sqrt{2\left(E_{0}-V\right)} \sqrt{g_{i j} d q^{i} d q^{j}}
$$

where we have an action integral over the generalized coordinates along all paths connecting $\gamma(a)$ and $\gamma(b)$.

\section{VARIATIONAL INTEGRATION SCHEME}

We use a variational integrator to simulate the dynamical systems given by (2). The numerical scheme is derived by considering a discrete version of the underlying variational principle. We construct a hybrid variational integrator in the sense that we switch between two discrete formulations (Hamilton's and Jacobi-Maupertuis principle) of the dynamical system and, thus, are able to efficiently handle high velocity regions.

Standard Variational Integrators. In the following we will briefly summarize the integrators derived by a discrete version of Hamilton's principle (1). We replace the state space $T Q$ by $Q \times Q$ (which is locally isomorphic to $T Q$ ) and consider the grid $\Delta t=\left\{t_{k}=k h \mid k=0, \ldots, N\right\}, N h=T$, where $N$ is a positive integer and $h$ the step size. We replace the path $q:[0, T] \rightarrow Q$ by a discrete path $q_{d}:\left\{t_{k}\right\}_{k=0}^{N} \rightarrow Q$, where we view $q_{k}=q_{d}(k h)$ as an approximation to $q(k h)$, [5].

We approximate the action integral in (1) of the exact solution by a discrete Lagrangian $L_{d}: Q \times Q \rightarrow \mathbb{R}$,

$$
L_{d}\left(q_{k}, q_{k+1}\right) \approx \int_{k h}^{(k+1) h} L(q(t), \dot{q}(t)) d t .
$$

The discrete version (7) of the Hamilton principle (1) requires the discrete path $\left\{q_{k}\right\}_{k=0}^{N}$ to satisfy

$$
\delta \sum_{k=0}^{N-1} L_{d}\left(q_{k}, q_{k+1}\right)=0
$$

for all variations $\left\{\delta q_{k}\right\}_{k=0}^{N}$ with $\delta q_{0}=\delta q_{N}=0$, which is equivalent to the discrete Euler-Lagrange equations

$$
D_{2} L_{d}\left(q_{k-1}, q_{k}\right)+D_{1} L_{d}\left(q_{k}, q_{k+1}\right)=0,
$$

for $k=1, \ldots, N-1$. The discrete left and right Legendre transformations $\mathbb{F}^{ \pm} L_{d}$ transform two points in configuration space to the momentum at each point as $\mathbb{F}^{-} L_{d}: Q \times Q \rightarrow T^{*} Q,\left(q_{k}, q_{k+1}\right) \mapsto\left(q_{k}, p_{k}\right)=\left(q_{k},-D_{1} L_{d}\left(q_{k}, q_{k+1}\right)\right)$ and $\mathbb{F}^{+} L_{d}: Q \times Q \rightarrow T^{*} Q,\left(q_{k}, q_{k+1}\right) \mapsto\left(q_{k+1}, p_{k+1}\right)=\left(q_{k+1}, D_{2} L_{d}\left(q_{k}, q_{k+1}\right)\right)$. Using these to initiate the algorithm for a given initial state, the iteration scheme (8) determines a discrete solution $q_{d}$ that is well known to have nice energy behavior and to preserve momentum maps and the symplectic form of the continuous solution (momentum-symplectic integrator [5]). Usually, a fixed time step $h$ is used, however, for systems with varying velocity regions an adaptive time-stepping strategy would be much more efficient. During the last years lots of work has been done in developing variational approaches, where the step size is chosen based on the potential energy or acceleration of the system (see e.g. [4]). Thereby, an extra nonlinear equation has to be solved to determine the new time step for each integration step. We also mention the work on explicit adaptive symplectic integrators [3] for separable Hamiltonian systems.

Rather than using a varying step size, in this paper, we want to make use of a discrete version of the JacobiMaupertuis principle. Using a constant time stepping, the resulting integration scheme is then applied to regions where smaller step sizes for the standard variational integrator would be required. Thus, instead of solving an extra equation for the step size in each integration step one identifies high velocity regions and then switches to another constant time stepping scheme with new parametrization. 
The Jacobi-Maupertuis Variational Integrator. We decide for one generalized coordinate being the reparametrization variable $q^{l}, q_{0}^{l} \leq q^{l} \leq q_{N}^{l}$ for the curve $\gamma$ such that $\gamma\left(q_{0}^{l}\right)=q_{0}, \gamma\left(q_{N}^{l}\right)=q_{N}$ with the coordinates also reparametrized by $q^{l}$ as $q^{i}\left(q^{l}\right), i=1, \ldots, n$. (By abuse of notation, we denote the curve by $\gamma$ independent on the specific parametrization.) Similar as before we consider the grid $\Delta q^{l}=\left\{q_{k}^{l}=q_{0}^{l}+k \theta, k=0, \ldots, N\right\}, N \theta=q_{N}^{l}-q_{0}^{l}$, where $\theta$ is the step size.

We replace the curve $\gamma:\left[q_{0}^{l}, q_{N}^{l}\right] \rightarrow Q$ by a discrete path $\gamma_{d}: \Delta q^{l} \rightarrow Q$, where $\gamma_{k}=\gamma_{d}\left(q_{0}^{l}+k \theta\right)$ is an approximation to $\gamma\left(q_{0}^{l}+k \theta\right)$. Let $q_{d}=\left\{\delta q_{k}\right\}_{k=0}^{N}$ be the discrete path defined by $q_{k}=\gamma_{k}$. Then, we approximate the integral in (5) by a discrete function $A_{d}: Q \times Q \rightarrow \mathbb{R}, A_{d}\left(q_{k}, q_{k+1}\right) \approx \int_{\gamma_{k, k+1}} \sqrt{2\left(E_{0}-V(q)\right)} \sqrt{g_{i j} d q^{i} d q^{j}}$, where $\gamma_{k, k+1}$ is the curve between the end points $\gamma_{k}$ and $\gamma_{k+1}$, e.g. we choose $A_{d}\left(q_{k}, q_{k+1}\right)=\sqrt{2\left(E_{0}-V\left(\frac{q_{k+1}+q_{k}}{2}\right)\right)} \sqrt{g_{i j}\left(q_{k+1}^{i}-q_{k}^{i}\right)\left(q_{k+1}^{j}-q_{k}^{j}\right)}$. The discrete version (9) of the Jacobi-Maupertius principle requires the discrete path $\left\{q_{k}\right\}_{k=0}^{N}$ to satisfy

$$
\delta \sum_{k=0}^{N-1} A_{d}\left(q_{k}, q_{k+1}\right)=0
$$

for all variations $\left\{\delta q_{k}\right\}_{k=0}^{N}$ with $\delta q_{0}=\delta q_{N}=0$. Note, that we have no discrete time parametrization in this principle. Thus, by fixing the grid $\Delta q^{l}$ we take variations only in the discrete coordinates $q_{k}^{i}, i \neq l$, which leads to the discrete equations

$$
D_{2} A_{d}\left(q_{k-1}, q_{k}\right)+D_{1} A_{d}\left(q_{k}, q_{k+1}\right)=0
$$

where $D$ is the gradient w.r.t. $\left(q_{k}^{1}, \ldots, q_{k}^{l-1}, q_{k}^{l+1}, \ldots, q_{k}^{n}\right)$ for $k=1, \ldots, N-1$. Equation (10) provides an iteration scheme (JM (Jacobi-Maupertuis) integrator) that determines the discrete path $q_{d}$, but gives no information about time parametrization. Once the system has been integrated from state $q_{k}$ to state $q_{k+1}$, the time can be reconstructed using

$$
\int_{q_{k}}^{q_{k+1}} \frac{d s}{\sqrt{2(E-V(q))}}=\int_{t_{k}}^{t_{k+1}} d t=t_{k+1}-t_{k}
$$

Switching Criteria. Note that the Jacobi-Maupertuis principle requires that $E>V(q)$ for the principle to be well posed. If the manifold $Q$ is compact and $E>\max V(q)$, then we are fine. But in more general cases, we expect any numerical integrator scheme based on Jacobi-Maupertuis (JM integrator) to be unstable when $E-V(q)$ is small, i.e., JM integrator works best when kinetic energy is large. On the other hand, traditional variational integrators require small step sizes when the kinetic energy is large. What we are proposing here is to combine these two approaches in a way that uses these integrators in regimes where they work best. As a criteria for the choice of the discrete update scheme (variational and JM integrator), we define a critical value of the kinetic energy $K_{c}$. As long as $K<K_{c}$, we use the standard variational integrator with the iteration scheme (8), which preserves momentum maps according to symmetries of the Lagrangian, the symplectic form and a modified energy. For $K \geq K_{C}$ we make use of the timeindependent JM integrator. Depending on the shape of the trajectory we decide for one generalized coordinate $q^{l}$ that locally parametrizes the curve and use the scheme (10) to determine the discrete trajectory within the high velocity region, for which the total energy of the system is preserved. During the JM integration it is also possible to change the parametrization coordinate. When we switch from the JM integrator back to the standard variational integrator we need to take care of the switching points. Using the last two points $q_{0}, q_{1}$ of the JM integrator, we compute the effective step size $\tilde{h}_{0}$ such that the discrete version of (11) holds true. Since the energy during the JM integration is preserved along the trajectory, it is desirable to start with the same discrete energy for the standard variational integration, i.e. we require $E_{0}=\frac{d}{d \tilde{h}} L_{d}\left(q_{1}, q_{2}\right)=: E_{d}\left(q_{1}, q_{2}\right)$, where $\tilde{h}$ is the step size resulting from the discrete time grid $\Delta t$ and $E_{d}: Q \times Q \rightarrow \mathbb{R}$ the discrete energy function. Solving the energy equation together with the scheme (8) in the first integration step, gives us the next discrete trajectory point $q_{2}$ as well as the new step size $\tilde{h}$ which is held fixed for the remaining iteration scheme. Note that by construction, the computed step size $\tilde{h}$ also guarantees that the discrete energy for the ensuing standard variational integrator is the same as the one of the JM integrator.

Note, that the implicit equation of the integration scheme (10) might be more difficult to solve than the standard discrete Euler-Lagrange equations (8). However, due to the parametrization by one generalized coordinate we have to solve for one less equation. Note also, that an extra equation to solve for a new step size $\tilde{h}$ is only required at switching points from the JM integrator back to standard variational integrator. Thus, rather than determining a new step size in each iteration one is faced with an appropriate choice of the critical kinetic energy value $K_{c}$ to identify high velocity regions. 

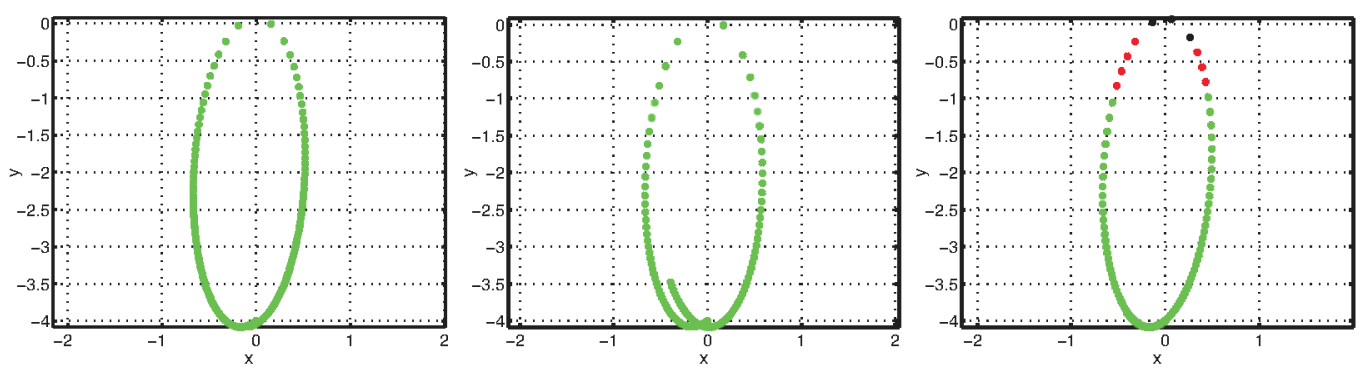

FIGURE 1. An elliptic Kepler orbit with initial condition $\left(x, y, p_{x}, p_{z}\right)(0)=(0,-4,-0.1,-0.1)$. Left: Constant time stepping $(h=0.005)$ variational integrator. Center: Constant time stepping $(h=0.01)$ variational integrator. Right: Hybrid integrator with step size $h=0.01$ for variational integrator part and step size $\theta=0.01$ for JM integrator part.

\section{NUMERICAL EXAMPLE}

We now illustrate the proposed integration scheme with the Kepler problem. Consider a satellite with mass $m$ which moves in the gravitational field of the Earth (mass $M$ ). In $2 \mathrm{~d}$ coordinates $q=(x, y)$, the Lagrangian of the system has the form $L(q, \dot{q})=\frac{1}{2} m\left(\dot{x}^{2}+\dot{y}^{2}\right)-V(q)$ with $V(q)=-\frac{m M}{\sqrt{x^{2}+y^{2}}}$. For this problem, the total energy $E=\frac{1}{2} m\left(\dot{x}^{2}+\dot{y}^{2}\right)+V(q)$ is a conserved quantity. The trajectories for the system are given by ellipses for $E<0$, parabolas for $E=0$, and hyperbolas for $E>0$. As an illustration, consider the case when $E<0$. For our simulation, we will assume $m=M=1$.

In Figure 1 (left) the simulation is done with a constant time stepping variational integrator with step size $h=0.005$. One can see more gaps in the upper high velocity region. The simulation shown in Figure 1 (center) uses a larger time step size $h=0.01$ for the same traditional variational integrator. The orbit no longer closes onto itself. Thus, the step size is not small enough to guarantee that the system lies in a constant energy surface in high velocity region. We now use our hybrid integrator to simulate the Kepler problem with critical kinetic energy value $K_{c}=0.75$. In Figure 1 (right), the green dots indicate the region in which we use a constant time stepping variational integrator with step size $h=0.01$, the red dots indicate the region where we use the Jacobi-Maupertuis (JM) integrator and where $\frac{d y}{d x} \geq 2$. The latter condition ensures that locally the curve is a graph with $y$ as a parameter. The black dots represent the region where we use the JM integrator but now $\frac{d y}{d x}<2$ and $x$ is chosen as a parameter for the curve with parameter step size $\theta=0.01$. Although we use the same time step as for the standard variational integrator, the trajectory nicely closes.

\section{CONCLUSION AND FUTURE WORK}

In this paper we proposed a new variational integrator that seems to be advantageous in handling high velocity regions. Based on a discrete version of the Maupertuis Principle of Least Action we created a hybrid variational integrator that switches between standard variational integration and a parameter independent discrete geodesic problem, where dependent on the trajectory shape an appropriate reparametrization can be chosen. We numerically illustrated the new integrator with the Kepler problem. Preliminary results indicate an improvement in the qualitative behavior of the integrator as opposed to just using the standard variational integrator. Ongoing work includes a detailed analysis of the energy and momentum behaviour and preservation of symplectic structures especially at switching points. We are also investigating links of the present work to the time-adaptive Hamilton-Pontryagin based variational integrators [4].

\section{REFERENCES}

1. V. I. Arnold, Mathematical Methods of Classical Mechanics, Springer, New York, 1989.

2. M. Biesiada, "The power of the Maupertuis-Jacobi principle - Dreams and reality". Chaos, Solitons \& Fractals 5(5), pp. 869-879 (1995).

3. S. Blanes and C. J. Budd, "Explicit Adaptive Symplectic (Easy) Integrators: A Scaling Invariant Generalisation of the Levi-Civita and KS Regularisations". Celestial Mechanics and Dynamical Astronomy 98(4), pp. 383-405 (2004).

4. L. Kharevych, P. Mullen, S. Leyendecker, Y. Tong, J. E. Marsden, and M. Desbrun, "Robust time-adaptive integrators for computer animation". In preparation (2009).

5. J. E. Marsden and M. West, "Discrete Mechanics and Variational Integrators". Acta Numerica 10, pp. 357-514 (2001).

6. G. Patrick and C. Cuell. Error analysis of variational integrators of unconstrained lagrangian systems. Numerische Mathematik, 2009, http://dx.doi.org/10.1007/s00211-009-0245-3. 